\title{
A SHARPENING OF MASLOV'S METHOD OF CHARACTERISTICS TO GIVE THE FULL ASYMPTOTIC SERIES*
}

\author{
By A. GORMAN AND R. WELLS (Pennsylvania State University)
}

1. Introduction. Maslov's method of characteristics uses a certain Hamiltonian flow to determine the first term in the asymptotic series solution of linear partial differential equations near turning points. In [1] an associated non-Hamiltonian flow was introduced to determine the full asymptotic series of the reduced Helmholtz equation. Here we note the same sharpening of Maslov's method extends to a much more general setting. We describe the sharpening for two independent space variables for all possible positions of the Lagrange manifold. For the case in which the Lagrange manifold cannot be parametrized by either space or momentum variables separately, another auxiliary flow is required to obtain the full asymptotic expansion. We determine this flow, then relate the each position of the Lagrange manifold to either the classical technique or a refined Maslov technique.

2. We assume that the wave-type equation

$$
\sum_{j=1}^{2} a_{j}(\bar{x}) \frac{\partial^{2} \psi}{\partial x_{j}^{2}}+\sum_{j=1}^{2} b_{j}(\bar{x}) \frac{\partial^{2} \psi}{\partial x_{j} \partial t}+\sum_{j=1}^{2} c_{j}(\bar{x}) \frac{\partial \psi}{\partial x_{j}}+\sum_{n=0}^{2} d_{n}(\bar{x}) \frac{\partial^{n} \psi}{\partial t^{n}}=0
$$

has an asymptotic solution - for brevity, near turning points of the highest order-of the form

$$
\psi(\bar{x})-\exp \{i \tau t\} \int A(\bar{x}, \bar{p}, \tau) \exp \left\{i \tau\left(\bar{x}^{\prime} \cdot \bar{p}-S(\bar{p})\right)\right\} d \bar{p}=O\left(\tau^{-\infty}\right)
$$

where $\tau$ is a large parameter and $S(\bar{p})$ is such that $\bar{x}-\nabla_{p} S(\bar{p})=0$ determines the Lagrange manifold of Maslov near the turning point [2]. Essentially, the Lagrange manifold is the set of points $(\bar{x}, \bar{p})$ at which is concentrated all the contribution to the asymptotic series of the integral. Notice that the integral alone is a full asymptotic solution of the "associated Helmholtz equation."

Carrying the differentiation (1) across the integral (2) determines

$$
\begin{gathered}
\int d \bar{p} \exp \{i \tau(\bar{x} \cdot \bar{p}-S(\bar{p}))\}\left\{(i \tau)^{2}\left[\sum_{j=1} a_{j} p_{j}^{2}+\sum_{j} b_{j} p_{j}+d_{2}\right] A\right. \\
+(i \tau)\left[\sum_{j=1} 2 a_{j} p_{j} \frac{\partial A}{\partial x_{j}}+\sum_{j} b_{j} \frac{\partial A}{\partial x_{j}}+\sum_{j} c_{j} p_{j} A+d_{1} A\right] \\
\left.+\left[\sum_{j} a_{j} \frac{\partial^{2} A}{\partial x_{j}^{2}}+\sum_{j} c_{j} \frac{\partial A}{\partial x_{j}}+d_{0} A\right]\right\}=0\left(\tau^{-\infty}\right) .
\end{gathered}
$$

* Received March 30, 1981. 
The coefficient of $(i \tau)^{2}$ is Maslov's Hamiltonian (and hence is zero) on the manifold [3, Chapter II]. Expanding the Hamiltonian

$$
\begin{aligned}
\sum_{j} a_{j}(\bar{x}) p_{j}^{2}+\sum_{j} b_{j}(\bar{x}) p_{j}+d_{2}(\bar{x})= & \sum_{j} a_{j}\left(\nabla_{p} S\right) p_{j}+\sum_{j} b_{j}\left(\nabla_{p} S\right) \\
& +d_{2}\left(\nabla_{p} S\right)+\sum_{j}\left(x_{j}-\frac{\partial S}{\partial p_{j}}\right) D_{j}=\sum_{j}\left(x_{j}-\frac{\partial S}{\partial p_{j}}\right) D_{j}
\end{aligned}
$$

where

$$
\bar{D}=\int_{0}^{1} \nabla_{x} H\left(t\left(\bar{x}-\nabla_{p} S(\bar{p})\right)+\nabla_{p} S(\bar{p}), \bar{p}\right) d t .
$$

with $H$ being Maslov's Hamiltonian. Substituting into (3), noting

$$
\begin{aligned}
& \left.\int \nabla_{p} \cdot[\exp \{i \tau(\bar{x} \cdot \bar{p}-S(\bar{p}))\} A \bar{D}\}\right] d \bar{p} \\
& \quad=\int \exp \{i \tau(\bar{x} \cdot \bar{p}-S(\bar{p}))\}\left\{i \tau A\left(\bar{x}-\nabla_{p} S(\bar{p})\right) \cdot \bar{D}+\bar{D} \cdot \nabla_{p} A+A \nabla_{p} \cdot \bar{D}\right\} d \bar{p},
\end{aligned}
$$

and taking the surface integral over a sufficiently large radius so that it vanishes, (3) becomes

$$
\begin{array}{r}
\int d \bar{p} \exp \{i \tau(\bar{x} \cdot \bar{p}-S(\bar{p}))\}\left\{( i \tau ) \left[-\sum_{j} D_{j} \frac{\partial A}{\partial p_{j}}-\sum_{j} A \frac{\partial D_{j}}{\partial p_{j}}+\sum_{j} 2 a_{j} p_{j} \frac{\partial A}{\partial x_{j}}+\sum_{j} b_{j} \frac{\partial A}{\partial x_{j}}\right.\right. \\
\left.\left.+\sum_{j} c_{j} p_{j} A+d_{1} A\right]+\sum_{j} a_{j} \frac{\partial^{2} A}{\partial x_{j}^{2}}+\sum_{j} c_{j} \frac{\partial A}{\partial x_{j}}+d_{0} A\right\}=O\left(\tau^{-\infty}\right) .
\end{array}
$$

Requiring

$$
\begin{aligned}
-\sum_{j} D_{j} \frac{\partial A}{\partial p_{j}}-\sum_{j} A \frac{\partial D_{j}}{\partial p_{j}}+\sum_{j} 2 a_{j} p_{j} \frac{\partial A}{\partial x_{j}}+\sum_{j} b_{j} \frac{\partial A}{\partial x_{j}}+\sum_{j} c_{j} p_{j} A+d_{1} A \\
+\frac{1}{i \tau}\left\{\sum_{j} \frac{\partial^{2} A}{\partial x_{j}^{2}}+\sum_{j} c_{j} \frac{\partial A}{\partial x_{j}}+d_{0} A\right\}=0
\end{aligned}
$$

in a neighborhood of the Lagrange manifold leads to a transport equation if we introduce the flow

$$
x_{j}^{\prime}=2 a_{j}(\bar{x}) p_{j}+b_{j}(\bar{x}), \quad p_{j}^{\prime}=-D_{j},
$$

where the primes indicate time derivatives [4]. Specifically, (5) holds in such a neighborhood if we allow the asymptotic series

$$
A(\bar{x}, \bar{p}, \tau)=\sum_{k=0}^{\infty} A_{k}(\bar{x}, \bar{p})(i \tau)^{-k}
$$

to evolve according to the transport equation

$$
\begin{aligned}
& A_{k}^{\prime}-\left[\sum_{j}\left(c_{j}(\bar{x}) p_{j}-\frac{\partial D_{j}}{\partial p_{j}}\right)+d_{1}\right] A_{k}+ \\
& \frac{1}{i \tau}\left[\sum_{j}\left(a_{j}(\bar{x}) \frac{\partial^{2} A_{k-1}}{\partial x_{j}^{2}}+c_{j}(\bar{x}) \frac{\partial A_{k-1}}{\partial x_{j}}\right)+d_{0} A_{k-1}\right]=0 .
\end{aligned}
$$


For example, applying the above to the Tricomi equation

$$
y \frac{\partial^{2} \psi}{\partial x^{2}}+\frac{\partial^{2} \psi}{\partial y^{2}}=0
$$

we obtain the flow

$$
\begin{gathered}
x=-\frac{2}{3} p_{x}(0)^{3} t^{3}+2 p_{x}(0) p_{y}(0) t^{2}+2 y(0) p_{x}(0) t+x(0), \quad y=-p_{x}(0)^{2} t^{2}+2 p_{y}(0) t+y(0), \\
p_{x}=p_{x}(0), \quad p_{y}=-p_{x}(0) t^{2}+2 p_{y}(0) .
\end{gathered}
$$

Depending on 'emitter conditions', that is, on the Lagrange manifold, the transport equations have many different solutions; one particularly neat family of solutions is given by $A_{k}=$ constant for all $k$, leading to a full asymptotic solution to the Tricomi equation of the form

$$
\psi(\bar{x})=\int \exp \left\{i \tau\left(x p_{x}+y p_{y}+p_{y}^{3} / 3 p_{x}^{2}\right)\right\} \alpha\left(p_{x}, p_{y}\right) d p_{x} d p_{y}
$$

where $\alpha(\bar{p})$ is any smooth function with compact support. The solution may be multiplied by a constant asymptotic series.

3. While the above treatment suffices for most wave-type equations, in some cases a mixed coordinate-momentum space Lagrange manifold is required. A simple but useful example is the Helmholtz equation

$$
\frac{\partial^{2} \psi}{\partial x^{2}}+\frac{\partial^{2} \psi}{\partial y^{2}}+\left(x^{4}+y\right) \frac{\partial^{2} \psi}{\partial t^{2}}=0
$$

with emitter conditions $\left(x, y, p_{x}, p_{y}\right)=\left(\theta, \theta^{2}, \theta^{2}, \theta\right)$. Analogous to (2) we assume an asymptotic solution of the form

$$
\psi(\bar{x})-\exp \{i \tau t\} \int A\left(x, y, p_{y}, \tau\right) \exp \left\{i \tau\left(y p_{y}-S\left(p_{y}, x\right)\right)\right\} d p_{y}=O\left(\tau^{-}\right)
$$

where $A\left(x, y, p_{y}, \tau\right)$ and all its derivatives are bounded, which determines the Lagrange manifold

$$
y=\partial S / \partial p_{y}, \quad p_{x}=-\partial S / \partial x .
$$

Carrying the differentiation (9) across the integral in (10) obtains

$$
\begin{aligned}
& \int d p_{y} \exp \left\{i \tau\left(y p_{y}-S\left(p_{y}, x\right)\right)\right\}\left\{(i \tau)^{2}\left[p_{y}^{2}+\left(\frac{\partial S}{\partial x}\right)^{2}-\left(x^{4}+y\right)\right] A\right. \\
&\left.+i \tau\left[2 p_{y} \frac{\partial A}{\partial y}-2 \frac{\partial S}{\partial x} \frac{\partial A}{\partial x}-\frac{\partial^{2} S}{\partial x^{2}} A\right]+\left[\frac{\partial^{2} A}{\partial x^{2}}+\frac{\partial^{2} A}{\partial y^{2}}\right]\right\}
\end{aligned}
$$

In this case Maslov's Hamiltonian becomes

$$
H=p_{y}^{2}+\left(\frac{\partial S}{\partial x}\right)^{2}-\left(x^{4}+y\right) .
$$


Expanding, we get

$$
p_{y}^{2}+\left(\frac{\partial S}{\partial x}\right)^{2}-\left(x^{4}+y\right)=p_{y}^{2}+\left(\frac{\partial S}{\partial x}\right)^{2}-\left(x^{4}+\frac{\partial S}{\partial p_{y}}\right)+\left(y-\frac{\partial S}{\partial p_{y}}\right) D=\left(y-\frac{\partial S}{\partial p_{y}}\right) D .
$$

where

$$
D=\int_{0}^{1} \frac{\partial}{\partial y} H\left(x, t\left(y-\frac{\partial S}{\partial p_{y}}\right)+\frac{\partial S}{\partial p_{y}}, \frac{\partial S}{\partial x}, p_{y}\right) d t .
$$

Now substituting into (12) and noting

$$
\begin{aligned}
\int \exp & \left\{i \tau\left(y p_{y}-S\right)\right\} A\left(y-\frac{\partial S}{\partial p_{y}}\right) D d p_{y} \\
= & \int \frac{\partial}{\partial p_{y}}\left[\exp \left\{i \tau\left(y p_{y}-S\right)\right\} A D\right] d p_{y}-\int \exp \left\{i \tau\left(y p_{y}-S\right)\right\}\left[D \frac{\partial A}{\partial p_{y}}+A \frac{\partial D}{\partial p_{y}}\right] d p_{y} \\
= & -\int \exp \left\{i \tau\left(y p_{y}-S\right)\right\}\left[D \frac{\partial A}{\partial p_{y}}+A \frac{\partial D}{\partial p_{y}}\right] d p_{y},
\end{aligned}
$$

(12) becomes

$$
\begin{aligned}
& \int d p_{y} \exp \left\{i \tau\left(y p_{y}-S\left(p_{y}, x\right)\right)\right\} \\
& \cdot\left[i \tau\left(-D \frac{\partial A}{\partial p_{y}}-A \frac{\partial D}{\partial p_{y}}+2 p_{y} \frac{\partial A}{\partial y}-2 \frac{\partial S}{\partial x} \frac{\partial A}{\partial x}-\frac{\partial^{2} S}{\partial x^{2}} A\right)+\frac{\partial^{2} A}{\partial x^{2}}+\frac{\partial^{2} A}{\partial y^{2}}\right] .
\end{aligned}
$$

Then introducing the flow

$$
x^{\prime}=-2 \frac{\partial S}{\partial x}, \quad y^{\prime}=2 p_{y}, \quad p_{x}^{\prime}=-4 x^{3}, \quad p_{y}^{\prime}=-D,
$$

leads to the transport equation

$$
\frac{d A_{k}}{d t}-A_{k} \frac{\partial D}{\partial p_{y}}-\frac{\partial^{2} S}{\partial x^{2}} A_{k}+\frac{1}{i \tau}\left(\frac{\partial^{2} A_{k-1}}{\partial x^{2}}+\frac{\partial^{2} A_{k-1}}{\partial y^{2}}\right)=0 .
$$

More generally, for wave-type equations (1) requiring a Lagrange manifold as in (11), the flow

$$
\begin{gathered}
x_{1}^{\prime}=b_{1}(\bar{x})-\left(2 a_{1}(\bar{x})+c_{1}(\bar{x})\right) \frac{\partial S}{\partial x_{1}}, \quad y_{1}^{\prime}=b_{2}(\bar{x})-\left(2 a_{2}(\bar{x})+c_{2}(\bar{x})\right) p_{2}, \\
p_{1}^{\prime}=-\partial H / \partial x_{1}, \quad p_{2}^{\prime}=-D
\end{gathered}
$$

determines the transport equation

$$
\frac{d A}{d t}-A \frac{\partial D}{\partial p_{2}}-a_{1}(\bar{x}) \frac{\partial^{2} S}{\partial x_{1}^{2}} A+d_{1}(\bar{x}) A+\frac{1}{i \tau}\left\{\sum_{j}\left[a_{j}(\bar{x}) \frac{\partial^{2} A}{\partial x_{j}^{2}}+c_{j}(\bar{x}) \frac{\partial A}{\partial x_{j}}\right]+d_{0}(\bar{x}) A\right\}=0 .
$$

For those cases requiring the Lagrange manifold

$$
x_{1}=\partial S / \partial p_{1}, \quad p_{2}=-\partial S / \partial x_{2},
$$


e.g., emitter conditions $\left(x, y, p_{x}, p_{y}\right)=\left(\theta, \theta^{2}, \theta, \theta^{2}\right)$, the subscripts on the coordinates and momenta in (16) and (17) are interchanged.

4. For a given linear hyperbolic differential equation, different 'emitter conditions' generate different Lagrange manifolds. While the classical technique often suffices, near turning points of the Lagrange manifold some version of Maslov's technique is required. For a particular problem, let $L$ be the Lagrange manifold and $\left(x_{1}^{0}, y_{1}^{0}, p_{1}^{0}, p_{2}^{0}\right) \in L$ be a turning point of the manifold. Because $L$ is a smooth two-dimensional manifold, there must exist two smooth functions, e.g., $f$ and $g$, such that $z_{1}=f\left(z_{3}, z_{4}\right)$ and $z_{2}=g\left(z_{3}, z_{4}\right)$, where $z_{1}, z_{2}, z_{3}, z_{4}$ is a suitable permutation of $x_{1}, x_{2}, p_{1}, p_{2}$. Since $L$ is a Lagrange manifold, the form $d x_{1} \wedge d p_{1}+d x_{2} \wedge d p_{2}$ restricted to the tangent space of $L$ must vanish. Thus we obtain a finite list of cases, namely:

1) $p_{1}=f(\bar{x}), p_{2}=g(\bar{x})$. In this case $f(\bar{x}) d x_{1}+g(\bar{x}) d x_{2}$ is exact and the point is not a turning point -in the vicinity the classical technique applies $[2,3]$.

2) $x_{1}=f(\bar{p}), x_{2}=g(\bar{p})$. In this case $f(\bar{p}) d p_{1}+g(\bar{p}) d p_{2}$ is exact. In this case we may apply the procedure of Sec. 2 .

3) $x_{1}=f\left(x_{2}, p_{1}\right), p_{2}=g\left(x_{2}, p_{1}\right)$. In this case $f\left(x_{2}, p_{1}\right) d p_{1}-g\left(x_{2}, p_{1}\right) d x_{2}$ is exact. In this case we may apply the procedure of Sec. 3 .

4) $x_{1}=f\left(x_{1}, p_{1}\right), p_{2}=g\left(x_{1}, p_{1}\right)$. In this case the Poisson bracket $\{f, g\}=-1$. It follows from a straight forward calculation that already (1), (2), or (3) holds.

Thus the extended Maslov technique applies to all possible cases of the second-order linear hyperbolic equation in two space and one time variables.

\section{REFERENCES}

[1] A. Gorman and R. Wells, A sharpening of Maslov's method for finding asymptotic solutions, Quart. Appl. Math. 38, 509-510(1981)

[2] J.-P. Seneor and R. Seneor, The Maslov-WKB method for the (AN-)harmonic oscillator, Arch. Rat. Mech. Anal. 61(5), 153-173(1976)

[3] V. Guillemin and S. Sternberg, Geometric asymptotics, American Mathematical Society, Providence, RI, 1977

[4] J. J. Duistermaat, Fourier integral operators, Courant Institute Lecture Notes, New York University, 1973, p. 18 